\title{
PERDAS NA PLATAFORMA DE CORTE DE UMA COLHEDORA COMBINADA DE GRÃOS NA COLHEITA DE SOJA
}

\author{
Tulio de Almeida Machadoํㅜ, Fábio Lúcio Santos², João Paulo Barreto Cunha ${ }^{3}$, Daniel Antônio da Cunha ${ }^{4}$, \\ Laysla Morais Coelho ${ }^{5}$
}

\section{RESUMO}

A soja (Glycine Max) é uma das espécies mais cultivadas no Brasil, com importante papel socioeconômico e produção estimada para a safra 2011/12 de 66,7 milhões de toneladas. Dentre os prejuízos no processo de colheita, as ocasionadas pelo mecanismo de corte da colhedora são significativas, correspondendo cerca de $85 \%$ das perdas totais durante a colheita mecanizada. Este trabalho foi desenvolvido com o objetivo de avaliar as perdas na plataforma de corte em diferentes configurações de operação. O estudo foi conduzido na fazenda Santa Rosa, localizada no município de Morrinhos-GO, em uma área de 58 ha irrigada por pivô central onde foi realizada a semeadura da variedade IGRA 516 RR. Como fonte de avaliação do estudo, foi utilizada uma colhedora da marca John Deere, modelo 1450. O experimento foi realizado em delineamento de blocos casualizados em esquema fatorial $2 \times 3$, com duas velocidades de operação e três rotações do molinete e sete repetições, totalizando 42 unidades experimentais. Os resultados mostram que as perdas na plataforma de corte foram significativas em função da interação dos fatores estudados, sendo os melhores resultados obtidos quando utilizado $30 \mathrm{rpm}$ na rotação do molinete e $5 \mathrm{~km} \mathrm{~h}^{-1}$ para a velocidade de avanço, totalizando perdas entre 25,8 e $54,6 \mathrm{~kg} \mathrm{ha}^{-1}$.

Palavras-chave: mecanização, colheita mecanizada e velocidade operacional

\section{ABSTRACT \\ LOSSES IN THE CUTTING DECK OF A COMBINED GRAIN HARVASTER DURING THE SOYBEAN HARVEST}

Soybean (Glycine max) is one of the most cultivated species in Brazil, with important socioeconomic role and estimated production for the 2011/12 crop of 66.7 million tons. Among the losses in the harvesting process, those caused by the cutting mechanism of the harvester are significant, representing about $85 \%$ of total losses during mechanical harvesting. This work was carried out to evaluate the losses in cutting deck on different operating configurations. The study was conducted at the Santa Rosa farm, located in the municipality of Morrinhos-GO, in an area of 58 ha irrigated by center pivot which was sowed variety of IGRA 516 RR. As a source of evaluation of the study, we used a harvester John Deere brand, model 1450. The experiment was conducted in a randomized block design in a $2 \times 3$ factorial, with two operating speeds and three rotations of the reel and seven repetitions, totaling 42 experimental units. The results show that losses in cutting deck were significant due to the interaction of the factors studied. The best results were obtained when it was used $30 \mathrm{rpm}$ rotation of the reel and $5 \mathrm{~km} \mathrm{~h}^{-1}$ for forward speed, totaling losses between 25,8 and $54,6 \mathrm{~kg} \mathrm{ha}^{-1}$.

Keywords: mechanization, mechanizated harvest, operating speed

Recebido para publicação em 14/08/2012. Aprovado em 25/09/2012.

1- Engenheiro Agrícola, Mestrando em Engenharia Agrícola, DEA/UFV, Viçosa-MG, machado.tulio@gmail.com

2- Engenheiro Agrícola, Professor Adjunto I, DEA/UFV, Viçosa-MG, fabio.ls@ufv.br

3- Engenheiro Agrícola, Doutorando em Engenharia Agrícola, DEG/UFLA, Lavras- MG, bcunha@posgrad.ufla.br

4- Estudante do curso de Engenharia Agrícola, UNUCET/UEG, Anápolis-GO, cunhaengenharia@hotmail.com

5- Estudante do curso de Agronomia, UFG, Goiânia-GO, layslamc@hotmail.com 


\section{INTRODUÇÃO}

No Brasil, a soja (Glycine max. (L.) Merril) é um dos produtos agrícolas mais importantes voltados para a exportação, com estimativa para a safra 2011/12 de 66,37 milhões de toneladas. As condições climáticas adversas causadas pelo fenômeno "La Niña" foram os responsáveis pelo resultado negativo da safra, sendo um dos fatores que ditam seu valor comercial juntamente com o aumento de consumo na alimentação humana e animal (CONAB, 2012).

Parte significativa das perdas ocorre durante a colheita mecanizada, reduzindo a produtividade e a rentabilidade da operação, acarretando prejuízos ao produtor, visto que se trata da operação final do processo produtivo. Dessa forma, a colheita mecanizada nos dias atuais preconiza a busca de maiores eficiências, principalmente por vir experimentando grande evolução tecnológica (TABILE et al., 2008).

De acordo com Carvalho Filho et al. (2005), as perdas na colheita são influenciadas, por fatores inerentes à cultura ou por fatores relacionados à colhedora, sendo ainda bastante comuns em várias regiões do Brasil. Apesar de não haver um diagnóstico sobre as perdas durante a colheita de soja, há relatos de lavouras que chegam ter perdas de até quatro sacas por hectare $\left(240 \mathrm{~kg} \mathrm{ha}^{-1}\right)$. No entanto, as perdas toleráveis durante a colheita de soja devem estar entre 42 e $60 \mathrm{~kg} \mathrm{ha}^{-1}$ (EMBRAPA, 2012).

Ferreira et al. (2007) constataram diferenças quanto às perdas quantitativas em função das velocidades de deslocamento de uma colhedora de fluxo axial com potência de $103 \mathrm{~kW}(140 \mathrm{cv})$, para as menores velocidades ocorreram maiores perdas. Mesquita et al. (2006) afirmam que as menores perdas na colheita são observadas para velocidades próximas à faixa de 4,5 a $5,5 \mathrm{~km} \mathrm{~h}^{-1}$. Em contrapartida Campos et al. (2005) e Magalhães et al. (2009), ao avaliarem a influência da velocidade operacional de colhedoras, não observaram diferenças significativas nas perdas quantitativas de grãos, por deficiência na altura do corte, no sistema de limpeza e nas perdas totais.

Em relação à idade de uso da colhedora e o nível de perdas na colheita, Mesquita et al. (2002) observaram que máquinas com mais de quinze anos de idade apresentaram perdas superiores em comparação com colhedoras de idade inferior a cinco anos. Entretanto, os autores observaram um elevado número de colhedoras com mais de quinze anos cujas perdas durante a colheita foram inferiores a $60 \mathrm{~kg} \mathrm{ha}^{-1}$, indicando que outros fatores, tais como o treinamento do operador, as condições da lavoura e a conservação da máquina tem grande influência sobre o nível de perdas.

Shanoski et al. (2011) concluíram que a velocidade de deslocamento não apresenta correlação significativa com as perdas na colheita e, aproximadamente, $75 \%$ das perdas ocorrem na plataforma de corte e $25 \%$ no sistema de trilha, separação e limpeza. Entretanto, Cunha \& Zandbergen (2007) afirmam que 80 a $85 \%$ das perdas na colheita mecanizada ocorrem pela ação dos mecanismos da plataforma de corte das colhedoras (molinete, barra de corte e condutor helicoidal), $12 \%$ ocasionadas pelos mecanismos internos (trilha, separação e limpeza) e 3\% causadas por deiscência natural.

Pinheiro Neto \& Gamero (2001) avaliaram perdas quantitativas durante o processo de colheita da soja e observaram que o mecanismo de corte e alimentação foi responsável por cerca de $61,9 \%$ a $88,6 \%$ das perdas totais, sendo que perdas maiores foram obtidas durante a colheita de áreas com plantas apresentando umidade inferior a $12,5 \%$.

Segundo Carvalho Filho et al. (2005), à medida que ocorre aumento de velocidade de deslocamento as perdas aumentam. Da mesma forma, Mesquita et al. (2002), ao comparar colhedoras mecanizadas em diversos estados brasileiros, encontraram diferenças para as perdas de grãos em função da velocidade de deslocamento, o que indica que as perdas quantitativas possuem comportamento diretamente proporcional ao acréscimo de velocidade de deslocamento das colhedoras.

Neste contexto, o presente trabalho foi desenvolvido com o objetivo avaliar as perdas na plataforma de corte de uma colhedora durante o processo de colheita de soja considerando diferentes velocidades de operação e rotações do molinete.

\section{REVENG}




\section{MATERIAL E MÉTODOS}

Este trabalho foi realizado na fazenda Santa Rosa, localizada no município de Morrinhos, estado de Goiás, em uma área experimental de 58 ha irrigada por pivô central. Foi utilizada a cultivar de soja (Glycine max L.) IGRA 516 RR, semeada na área experimental entre os dias 18/10/2011 e 21/10/2011, utilizando-se o sistema de plantio direto.

O processo de colheita aconteceu após 105 dias, sendo utilizada para a avaliação das perdas uma colhedora autopropelida da marca John Deere, modelo 1450, com sistema de trilha em fluxo radial, motor com $180 \mathrm{cv}$, capacidade de armazenamento de grãos colhidos de 5500 L e plataforma de corte de 19 pés $(5,8$ $\mathrm{m})$. No momento da colheita os grãos se encontravam com umidade de aproximadamente $15 \%$.

$\mathrm{O}$ experimento foi conduzido segundo delineamento em blocos casualizados em esquema fatorial $2 \times 3$, constituídos de duas velocidades de trabalho da colhedora $\left(5 \mathrm{e} 7 \mathrm{~km} \mathrm{~h}^{-1}\right)$ e três rotações do molinete na plataforma de corte $(20,30$ e 40 rpm), com sete repetições, totalizando 42 unidades experimentais. A velocidade de deslocamento da colhedora foi determinada pelo painel eletrônico da máquina, enquanto a velocidade do molinete foi determinada por meio da contagem do número de voltas no intervalo de tempo de um minuto (rpm).

Para a determinação das perdas naturais, foi posicionada uma armação com área de $2 \mathrm{~m}^{2}$ onde foram coletados grãos, plantas e vagens encontrados na superfície do solo, os quais não podem ser recolhidos pela plataforma de corte da colhedora e, posteriormente, pesados. Foram realizadas dez repetições, aleatoriamente, ao longo de toda a área experimental a ser colhida. De acordo com a equação 1 , as perdas naturais foram obtidas a partir da relação entre da massa de grãos perdidos e a área da armação empregada na avaliação.

$P_{n}=10 \frac{m_{n}}{A}$

em que

$\mathrm{P}_{\mathrm{n}}=$ Perda natural $\left(\mathrm{kg} \mathrm{ha}^{-1}\right)$;

$\mathrm{m}_{\mathrm{n}}=$ Massa de grãos perdidos naturalmente, na área da armação (g); e
$\mathrm{A}=$ Área da armação $\left(\mathrm{m}^{2}\right)$.

A operação da máquina nas parcelas foi realizada com as diferentes combinações de velocidades operacionais da máquina e as rotações do molinete da plataforma de corte. Dessa forma, as perdas na plataforma de corte foram determinadas por meio da contagem de grãos presentes na área do gabarito, onde para tal, a máquina era parada e, posteriormente, recuada em cinco metros. Assim a área colhida entre a plataforma de corte e a lavoura não colhida constituía uma parcela experimental.

Para a determinação das perdas na plataforma de corte da colhedora, foi empregado um gabarito com área de 3,5 $\mathrm{m}^{2}$. Dessa forma, todo material contido na área desse gabarito foi recolhido e, posteriormente ensacado, identificado e pesado.

A partir dos resultados das perdas de plataforma, foram subtraídas as perdas naturais, o que permitiu se estimar o valor real dos resultados, conforme apresentado na equação 2.

$P_{p c}=10 \frac{m_{p}}{A}-P_{n}$

em que

$\mathrm{P}_{\mathrm{pc}}=$ Perda na plataforma de recolhimento $\left(\mathrm{kg} \mathrm{ha}^{-1}\right)$; $\mathrm{m}_{\mathrm{p}}=$ Massa de grãos perdidos embaixo da plataforma de recolhimento, na área da armação (g);

$\mathrm{A}=$ Área da armação $\left(\mathrm{m}^{2}\right)$; e

$\mathrm{P}_{\mathrm{n}}=$ Perda natural $\left(\mathrm{kg} \mathrm{ha}^{-1}\right)$.

Os dados obtidos referentes às perdas na plataforma de corte da colhedora foram submetidos à análise de variância. O efeito dos fatores velocidade de deslocamento e rotação do molinete foram estudados por meio de análise de regressão, sendo os modelos escolhidos com base no coeficiente de determinação, na significância dos coeficientes de regressão, e na falta de ajustamento dos modelos utilizando-se o teste "T" a 5\%. Todas as análises estatísticas foram realizadas utilizandose o programa computacional SAS ${ }^{\circledR}$, versão 8.0.

\section{RESULTADOS E DISCUSSÃO}

No Quadro 1 são apresentados os resultados da 
análise de variância para as perdas na plataforma de corte durante o processo de colheita. Pode-se observar que a interação entre os fatores velocidade de deslocamento $(\mathrm{Vd})$ e rotação do molinete $(\mathrm{Rmo})$ foi significativa a $5 \%$ de probabilidade.

No Quadro 2 são apresentados os resultados para análise de regressão, a qual permitiu estudar $o$ efeito dos fatores velocidade de deslocamento e rotação do molinete sobre as perdas na plataforma de corte da colhedora durante a colheita mecanizada de soja. Observou-se que a falta de ajustamento do modelo selecionado foi não significativa, o que indica a adequabilidade do mesmo.

A equação 3 representa o modelo selecionado de acordo com a análise de regressão para avaliar o efeito da velocidade de deslocamento e rotação do molinete na perdas $\left(\mathrm{kg} \mathrm{ha}^{-1}\right)$ referentes a plataforma de corte.

$$
\begin{aligned}
& P P C=213,5-33,01 V_{d}-6,18 R_{m o}+1,38 V_{d} R_{m o} \\
& (\mathrm{R} 2=0,66)
\end{aligned}
$$

As diferentes velocidades de avanço foram fatores determinantes para perdas em colheita, pois com o aumento da velocidade de deslocamento, houve um aumento na quantidade de plantas tombadas e não colhidas, ocasionando perdas mais acentuadas. Mesquita et al. (2001) concluíram haver uma tendência de redução de perdas quando as colhedoras operam em velocidades de deslocamento inferiores a $7 \mathrm{~km} \mathrm{~h}^{-1}$.

Pode-se verificar na superfície de resposta da Figura 2 que as maiores perdas foram observadas para as maiores velocidades de deslocamento combinadas às maiores rotações do molinete, tendendo a maiores inclinações para o eixo que se refere à velocidade de rotação do molinete, fato explicado pelo coeficiente da equação que leva em consideração esse fator.

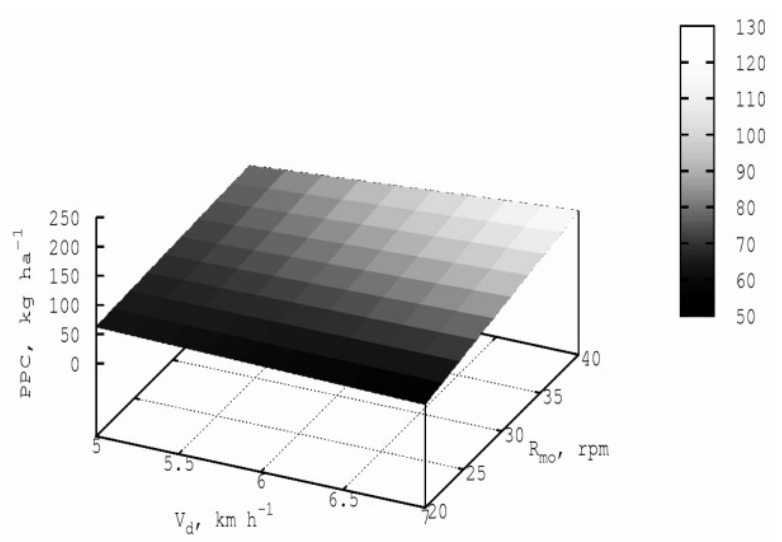

Figura 2. Superfície de resposta para perdas na plataforma de corte (PPC) de uma colhedora de soja em função da velocidade de deslocamento da máquina $\left(\mathrm{V}_{\mathrm{d}}\right)$ e da rotação do molinete $\left(\mathrm{R}_{\mathrm{mo}}\right)$.

Quadro 1. Resultado da análise de variância para a avaliação das perdas na plataforma de corte durante a operação de colheita de soja

\begin{tabular}{lccccc}
\hline \multicolumn{1}{c}{ FV } & GL & SQ & QM & F & P-valor \\
\hline Velocidade de deslocamento $(\mathrm{Vd})$ & 1 & 2849,39 & 2849,39 & -- & -- \\
Rotação do Molinete $\left(\mathrm{R}_{\mathrm{mo}}\right)$ & 2 & 15002,10 & 7501,05 & -- & -- \\
$\mathrm{V}_{\mathrm{d}} \times \mathrm{R}_{\mathrm{mo}}$ & 2 & 11629,29 & 5814,64 & $6,95^{*}$ & 0,0028 \\
Resíduo & 36 & 30123,00 & 836,75 & & \\
\hline Total & 41 & 59603,79 & & & \\
\hline
\end{tabular}

* significativo ao nível de $5 \%$ de probabilidade.

Quadro 2. Resultados da análise de regressão para estudar o efeito da velocidade de deslocamento e da rotação do molinete nas perdas da plataforma de corte durante a colheita mecanizada de soja

\begin{tabular}{lcc}
\hline \multicolumn{1}{c}{ FV } & GL & QM \\
\hline Regressão & 3 & $6702,81^{*}$ \\
Falta de ajustamento & 2 & $4686,16^{\text {ns }}$ \\
\hline Velocidade de deslocamento $\left(\mathrm{V}_{\mathrm{d}}\right)$, Rotação do Molinete $\left(\mathrm{R}_{\text {mo }}\right), \mathrm{V}_{\mathrm{d}} \mathrm{x} \mathrm{R}_{\mathrm{mo}}$ & 5 & 5896,15 \\
Resíduo & 36 & 836,75 \\
\hline Total & 41 & \\
\hline
\end{tabular}

* significativo ao nível de 5\% de probabilidade 
As perdas na plataforma foram diretamente influenciadas pela rotação do molinete. Observouse que as maiores perdas no sistema ocorreram para as maiores rotações do mecanismo, o que pode ter ocasionado a debulha das vagens por impacto, fazendo com que os grãos caíssem fora da plataforma de corte e não fossem recolhidos.

Em média, as maiores perdas foram encontradas para velocidade de deslocamento de $7 \mathrm{~km} \mathrm{~h}^{-1}$ e para a rotação do molinete de $40 \mathrm{rpm}$, correspondendo à $130 \mathrm{~kg} \mathrm{ha}^{-1}$. Em contrapartida, as menores perdas ocorreram quando a velocidade de deslocamento foi de $7 \mathrm{~km} \mathrm{~h}^{-1} \mathrm{e}$ a rotação do molinete de $20 \mathrm{rpm}$, correspondendo à $48,36 \mathrm{~kg}$ $\mathrm{ha}^{-1}$. Pode-se observar que com o aumento dos fatores estudados, velocidade de deslocamento e rotação do molinete, contribuiu para um aumento de $160,25 \%\left(79,8 \mathrm{~kg} \mathrm{ha}^{-1}\right)$ nas perdas referentes à plataforma de corte.

Mesquita (2001) correlacionou o aumento das perdas com maiores velocidades de operação da colhedora. Entretanto, Ferreira et al. (2007) observaram que não houveram perdas significativas com o aumento da velocidade.

$\mathrm{Na}$ Figura 3 é apresentado o efeito dos diferentes níveis relativos aos fatores velocidade de deslocamento e a rotação do molinete sobre as perdas na plataforma de corte. Pode-se verificar que a rotação do molinete influencia diretamente as perdas na plataforma, de tal forma que, quanto maior a rotação, maiores são as perdas. Também fica evidente que a velocidade de deslocamento da máquina acentua as perdas na plataforma.

No Quadro 3 são apresentados os dados estimados e observados relativos a perda na plataforma da colhedora em função da velocidade de avanço da máquina e da rotação do molinete.
A partir dos dados do Quadro 3, para a velocidade de $5 \mathrm{~km} \mathrm{~h}^{-1}$, observou-se que para a rotação do molinete em $30 \mathrm{rpm}$ proporcionou perdas médias de $49,36 \mathrm{~kg} \mathrm{ha}{ }^{-1}$, sendo superestimadas em $41,91 \%$ pelo modelo proposto. Resultados similares foram obtidos por Laette \& Bollan (2009), os quais concluíram que a rotação do molinete igual a $30 \mathrm{rpm}$ proporcionou menores perdas na plataforma de corte durante a colheita de soja. Entretanto, para a velocidade de $7 \mathrm{~km} \mathrm{~h}^{-1}$ e rotação do molinete de $20 \mathrm{rpm}$ foi observada o menor índice de perdas entre todas as combinações, cuja as perdas observadas obtiveram $48,36 \mathrm{~kg} \mathrm{ha}^{-1}$ e o valor observado foi de $52,03 \mathrm{~kg}$ $\mathrm{ha}^{-1}$, atingindo valores aceitáveis para perdas na plataforma e maior capacidade operacional maior para a operação de colheita.

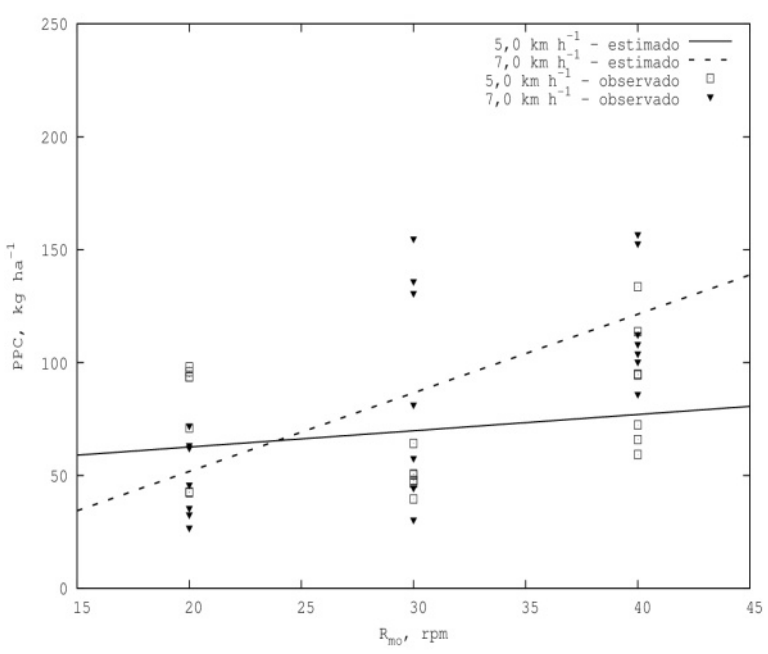

Figura 3. Corte na superfície de resposta para perdas na plataforma de corte (PPC) da rotação do molinete (Rmo).

Vale ressaltar que no presente trabalho, não há o somatório com as perdas proporcionadas

Quadro 3. Médias das perdas na plataforma $\left(\mathrm{kg} \mathrm{ha}^{-1}\right)$ em função da velocidade de avanço da colhedora $\left(\mathrm{km} \mathrm{h}^{-1}\right)$ e rotação do molinete (rpm)

\begin{tabular}{ccccc} 
& & \multicolumn{3}{c}{ Perdas na plataforma } \\
\hline \multirow{2}{*}{ Velocidade } & Rotação & Observado & Estimado & Desvio (\%) \\
\hline \multirow{2}{*}{5} & 20 & 76,76 & 62,85 & $-18,12$ \\
& 30 & 49,36 & 70,05 & 41,91 \\
\multirow{2}{*}{7} & 40 & 90,61 & 77,25 & $-14,74$ \\
\hline & 20 & 48,36 & 52,03 & 7,59 \\
& 30 & 90,81 & 86,83 & $-4,38$ \\
& 40 & 117,21 & 121,63 & 3,97 \\
\hline
\end{tabular}


pelos mecanismos internos, sendo que assim as perdas totais podem atingir valores maiores do que os aceitáveis pela EMBRAPA (60 $\left.\mathrm{kg} \mathrm{ha}^{-1}\right)$. Portanto, observou-se que a combinação entre o os fatores rotação do molinete e a maior velocidade de deslocamento influenciam diretamente nas perdas na plataforma. Esse resultado enfatiza a importância de estabelecer uma combinação adequada entre velocidade de deslocamento e a rotação do molinete para a minimização das perdas na plataforma de corte.

\section{CONCLUSÕES}

- As maiores perdas foram observadas para a velocidade de deslocamento de $7 \mathrm{~km} \mathrm{~h}^{-1} \mathrm{e}$ rotação do molinete de $40 \mathrm{rpm}$. As menores perdas foram encontradas para a velocidade de deslocamento de $5 \mathrm{~km} \mathrm{~h}^{-1}$ combinada a uma rotação do molinete de 30 rpm e para a velocidade de deslocamento de $7 \mathrm{~km} \mathrm{~h}^{-1}$ combinada a uma rotação do molinete de 20 rpm;

- A combinação entre a rotação do molinete e a velocidade de deslocamento são fundamentais para a minimização das perdas ocorridas na plataforma de corte durante o processo de colheita da soja; e

- A combinação de $7 \mathrm{~km} \mathrm{~h}^{-1}$ pode ser dita como ideal, pois mescla perdas aceitáveis com maior capacidade operacional da colhedora.

\section{REFERÊNCIAS BIBLIOGRÁFICAS}

CAMPOS, M.A.O.; SILVA, R.P.; CARVALHO FILHO, A.; MESQUITA, H.C.B.; ZABANI, S. Perdas na colheita mecanizada de soja no estado de Minas Gerais. Engenharia Agrícola, Jaboticabal, v.25, n.1, p.207-213, 2005.

CARVALHO FILHO, A., CORTEZ, J.W., SILVA R.P., ZAGO, M.S. Perdas na colheita mecanizada de soja no triângulo mineiro. Revista Nucleus, Ituverava, v.3, p.57-60, 2005.

CONAB - Companhia Nacional de Abastecimento. Acompanhamento de safra brasileira: grãos, décimo levantamento, julho 2012 / Companhia Nacional de Abastecimento. - Brasília: Conab, 2012.

CUNHA, J.P.A.R.; ZANDBERGEN, H.P. Perdas na colheita mecanizada da soja na região do Triângulo Mineiro e Alto Paranaíba, Brasil. Biosciense Journal, Uberlândia, v.23, n.4, p.6166, 2007.

EMBRAPA - Empresa Brasileira de Pesquisa Agropecuária. Centro Nacional de Pesquisa de Soja. Informativo eletrônico. Embrapa intensifica programa de perdas na colheita da soja. Ano 4; Edição 22 . Junho 2012.

FERREIRA I.C.; SILVA R.P.; LOPES A.; FURLANI C.E.A. Perdas quantitativas na colheita de soja em função da velocidade de deslocamento e regulagens no sistema de trilha. Engenharia na agricultura, Viçosa-MG, v.5, n.2, 141-150, 2007.

MAGALHÃES S.C.; OLIVEIRA B.C.; TOLEDO A.; TABILE R.A; SILVA R.P.; Perdas quantitativas na colheita mecanizada de soja em diferentes condições operacionais de duas colhedoras. Biosci. Journal, Uberlândia, v.25, n.5, p.43-48, 2009.

MESQUITA, C.M.; COSTA, N.P.; PEREIRA, J.E.; MAURINA, A.C.; ANDRADE, J.G.M. Caracterização da colheita mecanizada da soja no Paraná. Engenharia Agrícola, Jaboticabal, v.21, n.2, p.197-205, 2001.

MESQUITA, C.M.; COSTA, N.P.; PEREIRA, J.E.; MAURINA, A.C.; ANDRADE, J.G.M. Perfil da colheita mecânica da soja no Brasil: safra 1998/1999. Engenharia Agrícola, Jaboticabal, v.22, n.3, p.398-406, 2002.

MESQUITA, C.M.; HANNA, M.A.; COSTA, N.P. Crop and harvesting operation characteristics affecting field losses and physical qualities of soybeans - Part I. Applied Engineering in Agriculture, v.22, p.325-333, 2006.

PINHEIRO NETO, R.; GAMERO, C.A. Avaliação das Perdas Quantitativas de Grãos na Colheita 
de Soja. In: CONGRESSO BRASILEIRO DE ENGENHARIA AGRÍCOLA 25. Anais..., Foz do Iguaçu, p.13-16 Paraná. 2001.

SCHANOSKI.R.; RIGHI E.Z.; WERNER.V.; Perdas na colheita mecanizada de soja (Glycine max) no município de Maripá-PR. Revista Brasileira de Engenharia Agrícola e Ambiental, v 15, n.11, p 1206-1211, 2011.

TABILE, R.A.; TOLEDO, A.; SILVA, R.P.; FURLANI, C.E.A.; GROTTA, D.C.C.; CORTEZ, J.W. Perdas na colheita do milho em função da rotação do cilindro trilhador e umidade dos grãos. Revista Scientia Agrária, Curitiba, v.9, n.4, p.505-510, 2008. 